Article

\title{
Spectroscopic Parameter and Molecular Constant Investigations on Low-Lying States of BeF Radical
}

\author{
Zun Lue Zhu *, Qing Peng Song, Su Hua Kou, Jian Hua Lang and Jin Feng Sun \\ College of Physics \& Information Engineering, Henan Normal University, Xinxiang 453007, China; \\ E-Mails: shangen1987@163.com (Q.P.S.); coco13141@126.com (S.H.K.); \\ langjianhua87@163.com (J.H.L.); jfsun@htu.cn (J.F.S.) \\ * Author to whom correspondence should be addressed; E-Mail: zl-zhu@htu.cn; \\ Tel.: +86-373-332-6375; Fax: +86-373-332-6375.
}

Received: 12 December 2011; in revised form: 3 February 2012 / Accepted: 9 February 2012 / Published: 22 February 2012

\begin{abstract}
The potential energy curves (PECs) of $\mathrm{X}^{2} \Sigma^{+}, \mathrm{A}^{2} \Pi_{\mathrm{r}}$ and $\mathrm{B}^{2} \Sigma^{+}$states of $\mathrm{BeF}$ radical have been investigated using the complete active space self-consistent-field (CASSCF) method, followed by the highly accurate valence internally contracted multireference configuration interaction (MRCI) approach at the correlation-consistent basis sets, cc-pV5Z for Be and aug-cc-pV6Z for F. Based on the PECs of $\mathrm{X}^{2} \Sigma^{+}, \mathrm{A}^{2} \Pi_{\mathrm{r}}$ and $\mathrm{B}^{2} \Sigma^{+}$states, the spectroscopic parameters $\left(D_{e}, R_{e}, \omega_{e}, \omega_{e} \chi_{e}, \alpha_{e}\right.$ and $\left.B_{e}\right)$ have also been determined in the present work. With the PECs determined at the present level of theory, vibrational states have been predicted for each state when the rotational quantum number $J$ equals zero $(J=0)$. The vibrational levels, inertial rotation and centrifugal distortion constants are determined for the three states, and the classical turning points are also calculated for the $\mathrm{X}^{2} \Sigma^{+}$state. Compared with the available experiments and other theories, it can be seen that the present spectroscopic parameter and molecular constant results are more fully in agreement with the experimental findings.
\end{abstract}

Keywords: potential energy curve; dissociation energy; spectroscopic constant; molecular constant 


\section{Introduction}

Fluorides are a very important chemical species with broad applications in chemistry. The chemical property of fluorine is very lively and highly oxidized. In combination with other elements, resultant properties will be heat-resistant and difficult to erode by drugs and solvents. Fluorine is widely used in domestic appliances, office automation equipment, semiconductors, automobiles and other fields. Recently, with the development of calculation technology of quantum chemistry, more and more interest has been concentrated on the beryllium compounds [1-6]. As a simple fluoride compound, Beryllium Monofluoride (BeF) has been widely studied, both experimentally [7-11] and theoretically [12-21].

However, as can be seen in the literature, the experimental dissociation energies $D_{0}$ of BeF greatly differ from each other. For example, the value reported by Hildenbrand and Murad [7] in 1966 is of $5.85 \mathrm{eV}$ and the value determined by Farber and Srivastava [9] in 1974 is of $6.26 \mathrm{eV}$. Whereas this value collected in Reference [10] by Herzberg in 1950 is of $5.4 \mathrm{eV}$ and collected in Reference [11] by Huber and Herzberg in 1979 is of 6.26 or $5.85 \mathrm{eV}$. Obviously, it needs to be clarified urgently.

In theory, the spectroscopic parameters including the dissociation energy $D_{e}$ have been widely studied in the past several decades [12-21]. On the one hand, the $D_{e}$ values still show a wide variation. For example, Roach and Kuntz [12] investigated the $D_{e}$ in 1982, and gave a value of $3.94 \mathrm{eV}$. Partridge et al. [13] calculated the $D_{e}$ in 1984 with a value of $5.94 \mathrm{eV}$. On the other hand, it is still in question whether the potential barrier on the ground-state potential energy curve exists or not. For example, Roach [12] and Machado et al. [17] thought that the barrier obtained here, and the spectroscopic parameters are accurately determined. Finally, it is considered that numerically solving the radial Schrödinger equation is possible, but Marian [14] and Ornellas et al. [18] did not think so. Furthermore, some theoretical information [14,18,20,21] is available about the excited states of BeF. Some vibrational manifolds (such as vibrational levels, initial rotation and centrifugal distortion constants) have been reported in the literature, which have important applications in the vibrational transition calculations. All these aspects motivated us to perform the present investigations.

One of the purposes of this investigation is to determine the accurate potential energy curves of $\mathrm{X}^{2} \Sigma^{+}, \mathrm{A}^{2} \Pi_{\mathrm{r}}$ and $\mathrm{B}^{2} \Sigma^{+}$states for BeF radical, using the full valence complete active space self-consistent field method [22,23], followed by the highly accurate valence internally contracted multireference configuration interaction approach [24,25] in combination with the correlation-consistent basis sets [26-28], cc-pV5Z for Be and aug-cc-pV6Z for $\mathrm{F}$ atom. The spectroscopic parameters and vibrational manifolds are determined for these three states, using the obtained PECs of BeF radical, with the help of VIBROT module in MOLCAS 7.4 program package [29].

\section{Theoretical Approach}

We calculate the PECs of $\mathrm{X}^{2} \Sigma^{+}, \mathrm{A}^{2} \Pi_{\mathrm{r}}$ and $\mathrm{B}^{2} \Sigma^{+}$states of BeF by the CASSCF approach, followed by the MRCI calculations. Therefore, the full valence CASSCF is employed as the reference wavefunction for the MRCI calculations in the present work. For the PEC calculations, the MRCI theory has proven particularly successful [30-35]. The present calculations are carried out in MOLPRO 
2008.1 program package [36] with the largest correlation-consistent basis set, cc-pV5Z for Be and aug-cc-pV6Z for $\mathrm{F}$ atom.

$\mathrm{BeF}$ is of $\mathrm{C}_{\infty \mathrm{v}}$ point group symmetry. According to the molecular theory and the requirement of MOLPRO program package, it must be replaced by $\mathrm{C}_{2 v}$ symmetry with the order of the irreducible representations as $a_{1} / b_{1} / b_{2} / a_{2}$ in the calculations. In detail, eight molecular orbitals (MOs) are put into the active space, including four $a_{1}$, two $b_{1}$ and two $b_{2}$ symmetry MOs, which correspond to the $2 s$ shell of Be and $2 s 2 p$ shell of $\mathrm{F}$ atom. The rest of the electrons in the BeF radical are put into the closed-shell orbitals, including two $a_{1}$ symmetry MOs. When we use these MOs ( $\operatorname{six} a_{1}$, two $b_{1}$, two $b_{2}$ ) to calculate the PECs of the BeF radical, we find that the obtained PECs are smooth for all these basis sets over the present internuclear distance range.

In general, the PECs calculations are made at intervals of $0.02 \mathrm{~nm}$ over the internuclear distance range from 0.0522 to $2.0472 \mathrm{~nm}$. Near the equilibrium position, we chose the interval to be of $0.005 \mathrm{~nm}$ so that the properties of the PECs are displayed more clearly. With the PECs determined at the different basis sets, the spectroscopic parameters $\left(D_{e}, \omega_{e}, \omega_{e} \chi_{e}, \alpha_{e}, B_{e}\right.$ and $\left.D_{0}\right)$ are evaluated. By comparison with the experiments [7-11], we find that the best favorable spectroscopic parameter results can be obtained at the basis sets, cc-pV5Z for Be and aug-cc-pV6Z for F atom.

In order to take into consideration the relativistic effects on the spectroscopic parameters, the Douglas-Kroll one-electron integrals are used with the basis sets cc-pV5Z for Be and aug-cc-pV6Z for F. We notice that almost no accuracy improvements can be made for the spectroscopic parameters after considering the relativistic corrections. Therefore, vibrational manifold calculations are made at the PECs obtained at the non-relativistic condition.

\section{Results and Discussion}

\subsection{PECs of the BeF and Spectroscopic Parameters}

The PECs of BeF radical are shown in Figure 1. As shown in the figure, the $A^{2} \Pi_{r}$ curve and the $\mathrm{B}^{2} \Sigma^{+}$curve are all marginally repulsive at long range, but they do not converge. The $\mathrm{A}^{2} \Pi_{\mathrm{r}}$ state and the $\mathrm{X}^{2} \Sigma^{+}$state have the same dissociation channel $\mathrm{Be}\left({ }^{1} \mathrm{~S}_{\mathrm{g}}\right)+\mathrm{F}\left({ }^{2} \mathrm{P}_{\mathrm{u}}\right)$, which is different from $\mathrm{Be}\left({ }^{3} \mathrm{P}_{\mathrm{u}}\right)+\mathrm{F}\left({ }^{2} \mathrm{P}_{\mathrm{u}}\right)$ for the $\mathrm{B}^{2} \Sigma^{+}$state. During the course of the PEC investigation of the $\mathrm{X}^{2} \Sigma^{+}$state, the existence of the barrier was a hot topic and should be stressed here, however, that it is not the main goal of the present work. To illustrate the existence of the barrier of the PEC of the $\mathrm{X}^{2} \Sigma^{+}$state, a magnified image for the PEC of the $\mathrm{X}^{2} \Sigma^{+}$state has been shown in Figure 2. It has been found in our calculations that there is a small barrier in the curve of $\mathrm{X}^{2} \Sigma^{+}$state which has been found at the internuclear separation, $0.3372 \mathrm{~nm}$, and the barrier height is of $0.18 \mathrm{eV}$. A similar situation was also found by Roach [12] and Machado [17], but not by Marian [14] and Ornellas et al. [18]. Ornellas et al. [18] did not observe the small hump since the interval used was too large when they calculated the PEC. Marian [14] paid attention to calculating the spin-orbit coupling, and he considered 42 reference state functions to generate the CI wavefunction. In similarity with Reference [18], the interval was also too large in his calculations [14]. A wide barrier of $0.79 \mathrm{eV}$ has been found in the PEC of the $\mathrm{A}^{2} \Pi_{\mathrm{r}}$ state, similar to the value reported by Marian [14] and Ornellas et al. [18], $0.81 \mathrm{eV}$ and $0.79 \mathrm{eV}$, respectively. A similar feature has also been found for the $\mathrm{B}^{2} \Sigma^{+}$curve of the BeF radical. Near $0.18 \mathrm{~nm}$, the $\mathrm{B}^{2} \Sigma^{+}$state unfolds a sharp avoided 
crossing with the repulsive covalent state correlating with the dissociation channel $\mathrm{Be}\left({ }^{3} \mathrm{P}_{\mathrm{u}}\right)+\mathrm{F}\left({ }^{2} \mathrm{P}_{\mathrm{u}}\right)$. So the avoided crossing and the ionic character are responsible for the unusual shape of these potential curves.

Figure 1. Potential energy curves (PECs) of the BeF.

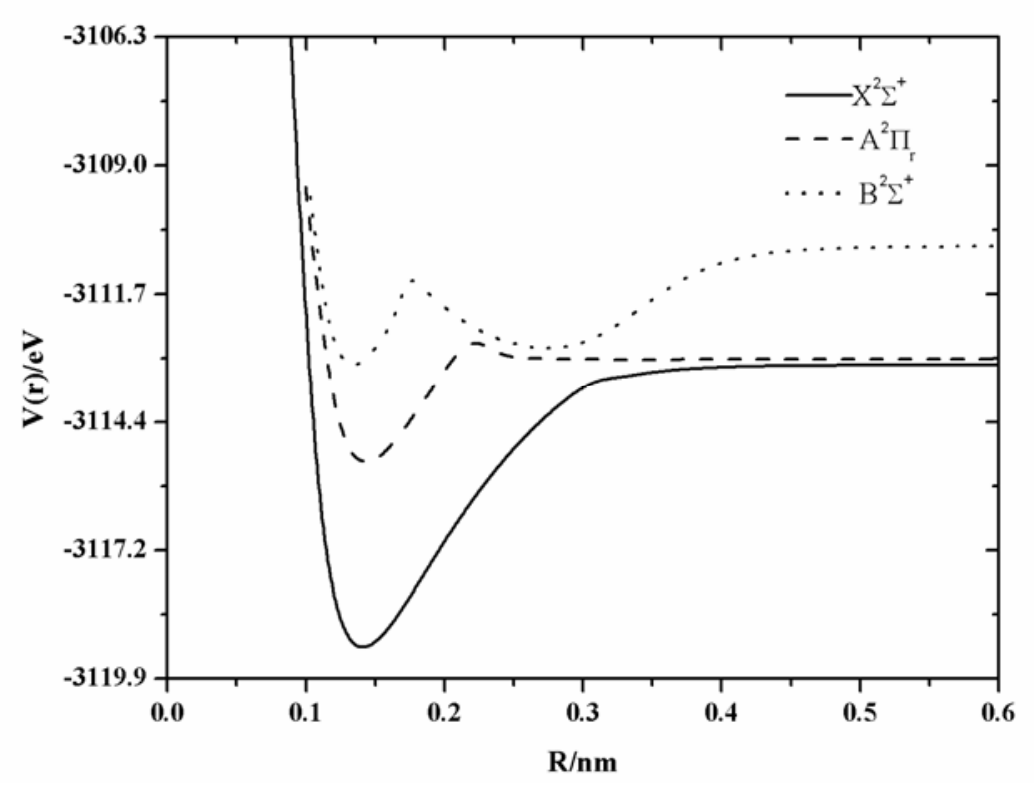

Figure 2. PEC of the $X^{2} \Sigma^{+}$state.

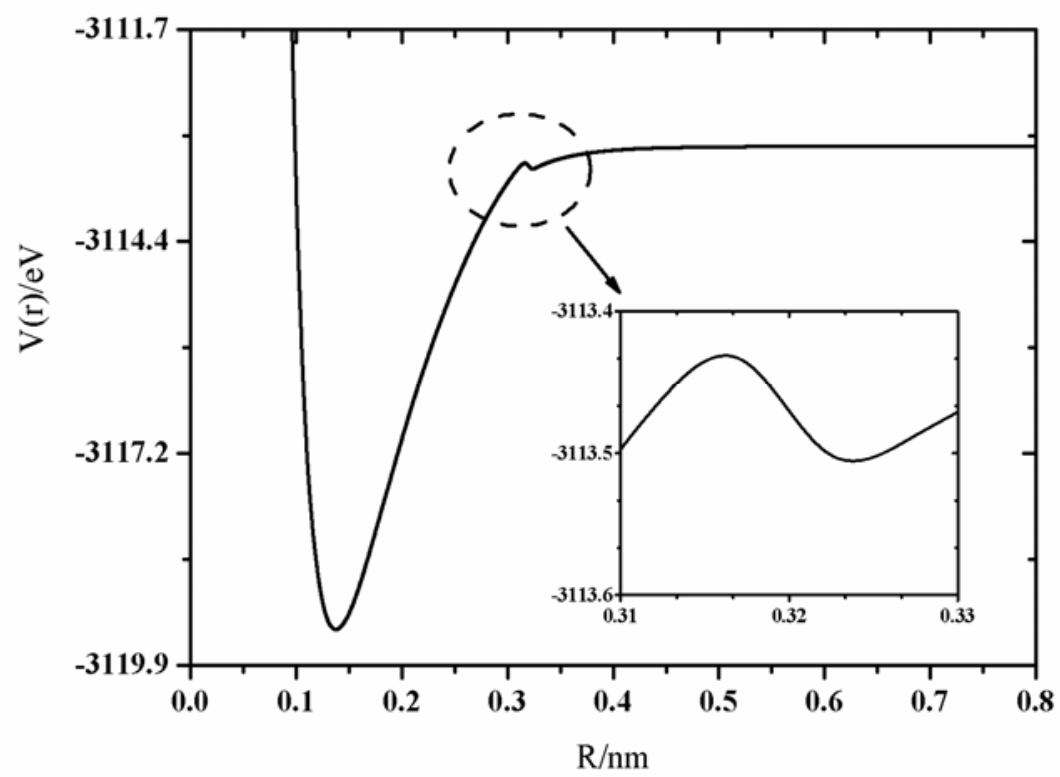

With the PECs determined, the spectroscopic parameters and molecular constants are evaluated with the VIBROT module in MOLCAS 7.4 program package. In order to conveniently compare the present results, we compiled the spectroscopic parameters together with the available experiments [7-11] and other theories [12-21] in Table 1 for the BeF radical. 
Table 1. Spectroscopic parameter comparison with available measurements and other theories for BeF radical.

\begin{tabular}{|c|c|c|c|c|c|c|c|}
\hline Source & $D_{e} / \mathrm{eV}$ & $R_{e} / \mathbf{n m}$ & $\omega_{e} / \mathbf{c m}^{-1}$ & $\omega_{e} \chi_{e} / \mathbf{c m}^{-1}$ & $B_{e} / \mathrm{cm}^{-1}$ & $\alpha_{e} / \mathbf{c m}^{-1}$ & $D_{0} / \mathrm{eV}$ \\
\hline \multicolumn{8}{|l|}{$\overline{\mathbf{X}^{2} \Sigma^{+}}$} \\
\hline This work & 6.22 & 0.1372 & 1236.12 & 9.11 & 1.4651 & 0.0175 & 6.14 \\
\hline Exp [7] & ---- & ---- & ---- & ---- & ---- & ---- & 5.85 \\
\hline Exp [9] & ---- & ---- & ---- & ---- & ---- & ---- & 6.26 \\
\hline $\operatorname{Exp}[10]$ & 5.48 & 0.13614 & 1265.6 & 9.12 & 1.4877 & 0.01685 & 5.4 \\
\hline $\operatorname{Exp}[11]$ & 6.34 or 5.93 & 0.1361 & 1247.36 & 9.12 & 1.4889 & 0.0176 & 6.26 or 5.85 \\
\hline Theory [12] & 3.94 & ---- & ---- & ---- & ---- & ---- & ---- \\
\hline Theory [13] & 5.94 & 0.135 & ---- & ---- & ---- & ---- & 5.86 \\
\hline Theory [14] & 5.5 & 0.1369 & 1258 & 8.8 & 1.472 & ---- & ---- \\
\hline \multicolumn{8}{|l|}{ Theory [15] } \\
\hline $\mathrm{SCF}$ & ---- & 0.1352 & 1280 & ---- & ---- & ---- & 5.88 \\
\hline $\mathrm{CI}(\mathrm{SD})$ & ---- & 0.1363 & 1250 & ---- & ---- & ---- & 5.94 \\
\hline Theory [16] & ---- & 0.13637 & 1250 & ---- & ---- & ---- & ---- \\
\hline Theory [17] & 6.00 & 0.13711 & 1265.7 & 9.26 & 1.469 & 0.0169 & 5.92 \\
\hline Theory [18] & 5.82 & 0.1369 & 1272.5 & 9.52 & 1.472 & 0.01695 & ---- \\
\hline Theory [19] & ---- & 0.137 & 1240 & ---- & ---- & ---- & --- \\
\hline $\begin{array}{c}\text { Theory [20] } \\
\mathbf{A}^{\mathbf{2}} \boldsymbol{\Pi}_{\mathbf{r}}\end{array}$ & ---- & 0.13531 & 1339.3 & 8.34 & ---- & ---- & $T_{e} / \mathrm{cm}^{-1}$ \\
\hline This work & 2.32 & 0.1397 & 1174.2 & 8.78 & 1.413 & 0.0170 & $32,343.9$ \\
\hline Exp [8] & ---- & 0.13935 & 1171.2 & ----- & 1.42024 & 0.0175 & 33,187 \\
\hline Exp [10] & ---- & 0.13941 & 1172.6 & 8.78 & 1.4186 & 0.0161 & $33,233.6$ \\
\hline Exp [11] & 1.81 or 2.22 & 0.13935 & 1154.67 & 8.78 & 1.42024 & 0.0175 & $33,233.6$ \\
\hline Theory [14] & 1.17 & 0.1387 & 1183 & 13.5 & 1.433 & ---- & 34,814 \\
\hline Theory [18] & 1.69 & 0.1395 & 1175.4 & 8.8 & 1.412 & 0.01713 & 33,974 \\
\hline Theory [20] & ---- & 0.1385 & 1226.8 & 7.42 & ---- & ---- & 34,902 \\
\hline $\begin{array}{l}\text { Theory [21] } \\
\mathbf{B}^{2} \boldsymbol{\Sigma}^{+}\end{array}$ & ---- & 0.1437 & 1116 & ---- & ---- & ---- & ---- \\
\hline This work & 2.60 & 0.1332 & 1351.1 & 12.7 & 1.554 & 0.0149 & 48,877 \\
\hline Exp [8] & ---- & 0.1335 & 1350.8 & ---- & 1.547 & ---- & 49,573 \\
\hline $\operatorname{Exp}[11]$ & $\begin{array}{c}2.51 \text { or } \\
2.977\end{array}$ & 0.1335 & 1350.8 & 12.6 & 1.547 & ---- & 49,570 \\
\hline Theory [14] & ---- & 0.1321 & 1503 & 13.1 & 1.580 & ---- & 50,844 \\
\hline
\end{tabular}

A number of theoretical investigations had been made on the spectroscopic parameters of the $X^{2} \Sigma^{+}$ state of the BeF radical. Partridge et al. [13] in 1984 carried out the $R_{e}, D_{e}$ and $D_{0}$ calculations using Hartree-Fock (HF) method and some empirical formulas with Slater-type orbital (STO) basis set. Although their calculational results are close to the experiments, the existing experimental values and some empirical formulas were used and only two spectroscopic parameters were evaluated in their investigations. In 1985, Marian [14] investigated the PEC using multireference doubles configuration interaction approach (MRDCI) method with the GTO DZP AO basis set. With the aid of PEC, they calculated several spectroscopic parameters. We can find that his $\omega_{e} \chi_{e}$ is slightly smaller than the present one when compared with the corresponding experiments, though his $R_{e}$ is in more agreement 
with the experiments than ours. Langhoff et al. [15] in 1986 calculated $R_{e}$ and $\omega_{e}$ by two methods. We find that their most favorable results were obtained by the configuration interaction (CI) approach. As shown in Table 1, it is believed that these results are the most accurate values so far, but only limited spectroscopic parameters are derived. Langhoff et al. [16] later evaluated the $R_{e}$ and $\omega_{e}$ by three approaches. By comparison with the experiments, we find that their most favorable results were obtained with the singles and doubles configuration interaction (SDCI) approach. Also, the values are in more agreement with the experiments when compared with the present ones. However, their investigations were not concerned with other spectroscopic parameters.

Later, Machado and Ornellas [17] in 1989 made the PEC calculations by multireference singles and doubles configuration interaction approach (MRSDCI) with the Gaussian sets $(5 s, 3 p)$ for Be and $(7 s, 4 p)$ for $\mathrm{F}$. As can be seen in Table 1 , their $\omega_{e}$ and $\omega_{e} \chi_{e}$ are too large when compared with the experiments. Three years later, Ornellas et al. [18] in 1992 made the PEC calculation for ground state. In the calculations, their approach is the MRSDCI and the basis sets are $(14 s 10 p 3 d 1 f) /[8 s 6 p 3 d 1 f]$ for $\mathrm{F}$ and $(11 s 6 p 1 d) /[6 s 4 p 1 d]$ for Be. By comparison with the present ones, it is not difficult to find that their $\omega_{e} \chi_{e}$ and $\omega_{e}$ are slightly larger than the present experiments. Recently, Li and Hamilton [19] in 2001 calculated the $R_{e}$ using density functional theory (DFT) and MØller-Plesset (MP2) methods with three basis sets. Their most favorable results were obtained by DFT (BH and HLYP) approach with $6-311+\mathrm{G}^{*}$ basis sets. However, they did not compute spectroscopic parameters apart from the $R_{e}$ and $\omega_{e}$. Recently, Pelegrini et al. [20] in 2005 performed some spectroscopic parameter calculations by the MRCI method with the aug-cc-pVQZ basis set. As tabulated in Table 1, their $\omega_{e} \chi_{e}$ is far from the measurements when compared with the present work. Furthermore, other important spectroscopic parameters (such as $B_{e}$ and $\alpha_{e}$ ) were not evaluated in their investigations.

For the $\mathrm{A}^{2} \Pi_{\mathrm{r}}$ state, Walker and Richards [21] performed the $R_{e}$ and $\omega_{e}$ calculations using two methods in 1967. We find that their optimal results were obtained by the configuration interaction (CI) approach. As shown in Table 1, their $\omega_{e}$ is slightly smaller than the experiment data and other important spectroscopic parameters were not evaluated in their investigations. In 1985, Marian [14] investigated the PEC using MRDCI method with a GTO DZP AO basis set, with the aid of PEC, they calculated several spectroscopic parameters. We can find that his $\omega_{e} \chi_{e}$ is too large and his $D_{\mathrm{e}}$ is too small when compared with the experiments. Furthermore, $\alpha_{e}$ was not evaluated in his investigations. Ornellas et al. [18] in 1992 made the PEC calculation for lowest-lying state. In the calculations, their approach is the MRSDCI and the basis sets are $(14 s 10 p 3 d 1 f) /[8 s 6 p 3 d 1 f]$ for F and $(11 s 6 p 1 d) /[6 s 4 p 1 d]$ for Be. By comparison, it is not difficult to find that their $\omega_{e} \chi_{e}$ and $\omega_{e}$ are slightly larger than the present experiments when compared with the present ones. Pelegrini et al. [20] also performed some spectroscopic parameter calculations for the $\mathrm{A}^{2} \Pi_{\mathrm{r}}$ state of the BeF radical using the MRCI method with the aug-cc-pVQZ basis set. As tabulated in Table 1, their $\omega_{e} \chi_{e}$ and $\omega_{e}$ are far from the available measurements when compared with our work.

For the $\mathrm{B}^{2} \Sigma^{+}$of $\mathrm{BeF}$ radical, few theoretical investigations have been made on the spectroscopic parameters. The earlier theoretical calculations were performed by Marian [14]. He investigated the PEC of $\mathrm{BeF}\left(\mathrm{B}^{2} \Sigma^{+}\right)$using MRDCI method with a GTO DZP AO basis set. We can find that his $\omega_{e}$ and $\omega_{e} \chi_{e}$ are too large when compared with the experiments. Furthermore, $D_{\mathrm{e}}$ and $\alpha_{e}$ were not evaluated in his investigations. 
According to the above analysis and discussion, on the whole, the spectroscopic parameters obtained in the present work have improved when compared with previous theoretical results. For example, for the $\mathrm{X}^{2} \Sigma^{+}$state, the spectroscopic parameters, $\omega_{e} \chi_{e}, \alpha_{e}, \omega_{e}, B_{e}$ and $R_{\mathrm{e}}$, deviate from the experiments [11] only by $0.11 \%, 0.57 \%, 0.90 \%, 1.60 \%$ and $0.81 \%$, respectively. For the $\operatorname{BeF}\left(\mathrm{A}^{2} \Pi_{\mathrm{r}}\right)$, the spectroscopic parameters, $\omega_{e} \chi_{e}, \alpha_{e}, \omega_{e}, B_{e}$ and $R_{\mathrm{e}}$, deviate from the experiments [11] only by $0.00 \%, 2.86 \%, 1.69 \%$, $0.51 \%$ and $0.25 \%$, respectively.

As for the dissociation energy $D_{e}$ of $\mathrm{BeF}\left(\mathrm{X}^{2} \Sigma^{+}\right)$, it shows a wide variation. Roach and Kuntz [12] in 1982 made valence-bond (VB) calculations on the $\mathrm{BeF}\left(\mathrm{X}^{2} \Sigma^{+}\right)$radical, and they obtained the value to be $3.94 \mathrm{eV}$. But they claimed that their VB calculations are not accurate enough to deduce the accurate value of $D_{e}$ in Reference [12]. Partridge et al. [13] calculated the $D_{0}$ with empirical formula and obtained the direct value of $D_{0}$ to be $5.86 \mathrm{eV}$, and also gave the estimate result of $5.91 \mathrm{eV}$. The precision of the method is slightly lower than this work. Marian [14] investigated the PEC using MRDCI method with a GTO DZP AO basis set. They obtained $D_{e}$ of $5.5 \mathrm{eV}$, however, he thought that the value is a little small. Langhoff et al. [15] calculated the $D_{\mathrm{e}}$ by the SCF method. As we know, the method is too simple so that the $D_{e}$ result they obtained is not very credible. Machado and Ornellas [17] calculated the $D_{\mathrm{e}}$ by MRSDCI approach with the Gaussian sets $(5 \mathrm{~s}, 3 \mathrm{p})$ for Be and $(7 \mathrm{~s}, 4 \mathrm{p})$ for F. Ornellas et al. [18] computed the $D_{\mathrm{e}}$ by the MRSDCI method and the basis sets are $(11 \mathrm{~s} 6 \mathrm{p} 1 \mathrm{~d}) /[6 \mathrm{~s} 4 \mathrm{p} 1 \mathrm{~d}]$ for Be and $(14 s 10 p 3 d 1 f) /[8 s 6 p 3 d 1 f]$ for $\mathrm{F}$. The basis sets they used are very small. Therefore, their values are less accurate. In the present work, the PEC of $\operatorname{BeF}\left(\mathrm{X}^{2} \Sigma^{+}\right)$is computed using the highly accurate MRCI approach with the large basis sets, cc-pV5Z for Be and aug-cc-pV6Z for F. With the aid of PEC, the $D_{e}$ is determined to be $6.22 \mathrm{eV}$, which should be relatively close to the true value.

In this paper, we also calculate the $\Delta T_{e}$ of the $A^{2} \Pi_{\mathrm{r}}$ state is of $32,343.9 \mathrm{~cm}^{-1}$, while the value obtained by Marian [14], Ornellas et al. [18] and Pelegrini et al. [20] to be $34,814 \mathrm{~cm}^{-1}, 33,974 \mathrm{~cm}^{-1}$ and $34,902 \mathrm{~cm}^{-1}$, respectively. And the $\Delta T_{e}$ of the $\mathrm{B}^{2} \Sigma^{+}$state is also calculated, and the value is of $48,877 \mathrm{~cm}^{-1}$, the data reported by Marian [14] to be $50,844 \mathrm{~cm}^{-1}$.

It is widely recognized that the accuracy of the spectroscopic parameters calculations mainly depends on the scanned results for the PEC of the electronic state by using CASSCF AND MRCI approach. The scanned results of the electronic state are related to the choice of the active space for a CASSCF and of the basis sets. For BeF radical, the each electronic state possesses different bonding orbitals at various internuclear sparations [14]. In order to obtain more accurate calculational results of PECS of BeF radical, eight molecular orbitals, including four $a_{1}$, two $b_{1}$ and two $b_{2}$ symmetry MOs, are put into the active space, and the rest of the electrons in the BeF radical are put into two $a_{1}$ symmetry closed-shell orbitals, which differ from Reference [20]. In addition, the appropriate choices of the basis sets and the calculational interval in the CASSCF calculation also conduce to the accurate calculational results. So we have reasons to believe that the present results are reliable.

\subsection{Vibrational Manifolds}

Based on the reliable PECs of the $\mathrm{X}^{2} \Sigma^{+}, \mathrm{A}^{2} \Pi_{\mathrm{r}}$ and $\mathrm{B}^{2} \Sigma^{+}$states, we determine their vibrational levels, inertial rotation and centrifugal constants when $J=0$. And we also compute classical turning points for the ground state. Owing to the length limitation of the paper, we only tabulate some of these results for the vibrational states in Tables 2-7. To the best of our knowledge, no experimental data of molecular 
constants have been found in the literature, except several groups of theoretical results. But according to the remarkable agreement between the present spectroscopic parameters and the available experiments and the excellent accordance between the theoretical and the corresponding RKR data, we have reasons to believe that the results collected in Tables 2-7 are accurate.

Table 2. Comparison of the present and other theoretical vibrational level spacings (in $\left.\mathrm{cm}^{-1}\right), G(v+1)-G(v)$.

\begin{tabular}{cccccccccc}
\hline $\boldsymbol{v}$ & This work & Ref. [17] & Ref. [8] & Ref. [18] & $\boldsymbol{v}$ & This work & Ref. [17] & Ref. [8] & Ref. [18] \\
\hline 0 & 1254.0 & 1255.6 & 1254.5 & 1247.2 & 14 & 1021.1 & 1024.4 & 1009.3 & 1003.7 \\
1 & 1236.4 & 1239.5 & 1233.6 & 1229.0 & 15 & 1005.4 & 1007.7 & 993.0 & 987.4 \\
2 & 1218.9 & 1221.6 & 1215.4 & 1210.8 & 16 & 989.8 & 991.5 & 997.0 & \\
3 & 1201.5 & 1202.9 & 1197.5 & 1192.8 & 17 & 947.3 & 975.7 & 961.4 & \\
4 & 1184.5 & 1184.8 & 1179.7 & 1175.0 & 18 & 958.8 & 960.4 & & \\
5 & 1167.5 & 1167.7 & 1162.3 & 1157.4 & 19 & 943.5 & 945.6 & & \\
6 & 1150.7 & 1151.9 & 1144.5 & 1139.5 & 20 & 928.2 & 931.3 & & \\
7 & 1134.0 & 1136.6 & 1126.8 & 1122.2 & 21 & 912.9 & 917.5 & & \\
8 & 1117.5 & 1121.4 & 1109.4 & 1104.9 & 22 & 897.8 & 904.0 & & \\
9 & 1101.2 & 1106.2 & 1092.1 & 1086.8 & 23 & 882.6 & 890.8 & & \\
10 & 1084.9 & 1090.6 & 1075.1 & 1070.6 & 24 & 867.5 & 877.8 & & \\
11 & 1068.8 & 1074.6 & 1058.5 & 1053.7 & 25 & 852.5 & 865.1 & & \\
12 & 1052.8 & 1058.2 & 1042.0 & 1036.9 & 26 & 837.5 & & & \\
13 & 1036.9 & 1041.3 & 1025.6 & 1020.2 & 27 & 822.5 & & & \\
$\mathrm{G}(0)$ & 634.1 & 634.4 & 635.0 & ---- & & & & & \\
\hline
\end{tabular}

Table 3. Vibrational levels and classical turning points for $\operatorname{BeF}\left(\mathrm{X}^{2} \Sigma^{+}\right)$radical when $J=0$ at the MRCI level of theory.

\begin{tabular}{cccccccc}
\hline $\boldsymbol{v}$ & $\boldsymbol{G}(\boldsymbol{v}) / \mathbf{c m}^{-\mathbf{1}}$ & $\boldsymbol{R}_{\mathbf{m i n}} / \mathbf{n m}$ & $\boldsymbol{R}_{\mathbf{m a x}} / \mathbf{n m}$ & $\boldsymbol{v}$ & $\boldsymbol{G}(\boldsymbol{v}) / \mathbf{c m}^{-\mathbf{1}}$ & $\boldsymbol{R}_{\mathbf{m i n}} / \mathbf{n m}$ & $\boldsymbol{R}_{\mathbf{m a x}} / \mathbf{n m}$ \\
\hline 0 & 634.075 & 0.13102 & 0.14423 & 38 & $36,940.270$ & 0.10274 & 0.25274 \\
1 & 1888.092 & 0.12696 & 0.14998 & 39 & $37,598.068$ & 0.10253 & 0.25580 \\
2 & 3124.450 & 0.12438 & 0.15427 & 40 & $38,240.767$ & 0.10232 & 0.25890 \\
3 & 4343.333 & 0.12240 & 0.15798 & 41 & $38,868.312$ & 0.10212 & 0.26207 \\
4 & 5544.919 & 0.12077 & 0.16135 & 42 & $39,480.674$ & 0.10193 & 0.26530 \\
5 & 6729.378 & 0.11937 & 0.16450 & 43 & $40,077.768$ & 0.10175 & 0.26861 \\
6 & 7896.876 & 0.11815 & 0.16751 & 44 & $40,659.536$ & 0.10157 & 0.27199 \\
7 & 9047.568 & 0.11705 & 0.17039 & 45 & $41,225.903$ & 0.10139 & 0.27545 \\
8 & $10,181.605$ & 0.11606 & 0.17319 & 46 & $41,776.789$ & 0.10123 & 0.27899 \\
9 & $11,299.129$ & 0.11516 & 0.17592 & 47 & $42,312.104$ & 0.10107 & 0.28265 \\
10 & $12,400.279$ & 0.11432 & 0.17860 & 48 & $42,831.750$ & 0.10092 & 0.28639 \\
11 & $13,485.183$ & 0.11355 & 0.18123 & 49 & $43,335.622$ & 0.10077 & 0.29026 \\
12 & $14,553.965$ & 0.11283 & 0.18383 & 50 & $43,823.604$ & 0.10063 & 0.29425 \\
13 & $15,606.742$ & 0.11216 & 0.18641 & 51 & $44,295.572$ & 0.10049 & 0.29837 \\
14 & $16,643.623$ & 0.11153 & 0.18896 & 52 & $44,751.390$ & 0.10037 & 0.30263 \\
15 & $17,664.713$ & 0.11094 & 0.19150 & 53 & $45,190.911$ & 0.10024 & 0.30706 \\
16 & $18,670.109$ & 0.11037 & 0.19400 & 54 & $45,613.978$ & 0.10020 & 0.31166 \\
\hline
\end{tabular}


Table 3. Cont.

\begin{tabular}{llllllll}
\hline 17 & $19,659.902$ & 0.10984 & 0.19655 & 55 & $46,020.417$ & 0.10010 & 0.31646 \\
18 & $20,634.177$ & 0.10934 & 0.19907 & 56 & $46,410.044$ & 0.09990 & 0.32147 \\
19 & $21,593.013$ & 0.10886 & 0.20158 & 57 & $46,782.655$ & 0.09980 & 0.32673 \\
20 & 22536.484 & 0.10839 & 0.20411 & 58 & 47138.033 & 0.09971 & 0.33226 \\
21 & 23464.657 & 0.10796 & 0.20663 & 59 & 47475.938 & 0.09961 & 0.33809 \\
22 & 24377.591 & 0.10754 & 0.20916 & 60 & 47796.109 & 0.09953 & 0.34428 \\
23 & 25275.345 & 0.10715 & 0.21171 & 61 & 48098.263 & 0.09945 & 0.35088 \\
24 & 26157.965 & 0.10677 & 0.21426 & 62 & 48382.086 & 0.09937 & 0.35794 \\
25 & 27025.498 & 0.10639 & 0.21683 & 63 & 48647.232 & 0.09930 & 0.36555 \\
26 & 27877.980 & 0.10605 & 0.21943 & 64 & 48893.320 & 0.09924 & 0.37383 \\
27 & 28715.446 & 0.10571 & 0.22204 & 65 & 49119.923 & 0.09918 & 0.38289 \\
28 & 29537.922 & 0.10539 & 0.22467 & 66 & 49326.559 & 0.09912 & 0.39295 \\
29 & 30345.429 & 0.10508 & 0.22732 & 67 & 49512.685 & 0.09907 & 0.40426 \\
30 & 31137.985 & 0.10478 & 0.23001 & 68 & 49677.674 & 0.09903 & 0.41721 \\
31 & 31915.599 & 0.10449 & 0.23272 & 69 & 49820.797 & 0.09899 & 0.43242 \\
32 & 32678.277 & 0.10421 & 0.23546 & 70 & 49941.183 & 0.09896 & 0.45089 \\
33 & 33426.018 & 0.10394 & 0.23824 & 71 & 50037.765 & 0.09894 & 0.47456 \\
34 & 34158.817 & 0.10368 & 0.24106 & 72 & 50109.176 & 0.09892 & 0.50785 \\
35 & 34876.662 & 0.10344 & 0.24391 & 73 & 50153.519 & 0.09891 & 0.56546 \\
36 & 35579.535 & 0.10319 & 0.24681 & 74 & 50165.999 & 0.09896 & 0.65321 \\
37 & 36267.414 & 0.10297 & 0.24975 & & & & \\
\hline
\end{tabular}

Table 4. Rotational constants for $\mathrm{BeF}\left(\mathrm{X}^{2} \Sigma^{+}\right)$radical.

\begin{tabular}{|c|c|c|c|c|c|c|}
\hline \multirow{2}{*}{$v$} & \multicolumn{3}{|c|}{$B_{v} / \mathbf{c m}^{-1}$} & \multicolumn{3}{|c|}{$D_{v} / \mathrm{cm}^{-1}$} \\
\hline & This work & Theory $^{[17]}$ & Theory $^{[18]}$ & This work & Theory $^{[17]}$ & Theory $^{[18]}$ \\
\hline 0 & 1.466 & 1.4640 & 1.463 & 7.755 & 7.865 & 7.367 \\
\hline 1 & 1.440 & 1.4471 & 1.444 & 7.710 & 7.888 & 7.630 \\
\hline 2 & 1.423 & 1.4297 & 1.427 & 7.667 & 7.827 & 7.647 \\
\hline 3 & 1.407 & 1.4132 & 1.411 & 7.623 & 7.820 & 7.419 \\
\hline 4 & 1.390 & 1.3971 & 1.394 & 7.581 & 7.817 & 7.366 \\
\hline 5 & 1.375 & 1.3808 & 1.377 & 7.540 & 7.728 & 6.406 \\
\hline 6 & 1.359 & 1.3641 & 1.361 & 7.498 & 7.669 & 7.506 \\
\hline 7 & 1.343 & 1.3475 & 1.345 & 7.459 & 7.695 & 6.988 \\
\hline 8 & 1.327 & 1.3310 & 1.329 & 7.420 & 7.630 & 7.366 \\
\hline 9 & 1.311 & 1.3146 & 1.313 & 7.383 & 7.605 & 7.688 \\
\hline 10 & 1.296 & 1.2984 & 1.297 & 7.346 & 7.555 & 6.406 \\
\hline 11 & 1.280 & & & 7.310 & & \\
\hline 12 & 1.265 & & & 7.277 & & \\
\hline 13 & 1.250 & & & 7.245 & & \\
\hline 14 & 1.234 & & & 7.214 & & \\
\hline 15 & 1.219 & & & 7.184 & & \\
\hline 16 & 1.204 & & & 7.157 & & \\
\hline 17 & 1.189 & & & 7.130 & & \\
\hline 18 & 1.174 & & & 7.107 & & \\
\hline 19 & 1.159 & & & 7.084 & & \\
\hline 20 & 1.145 & & & 7.064 & & \\
\hline
\end{tabular}


Table 5. The centrifugal distortion constants for the $\operatorname{BeF}\left(\mathrm{X}^{2} \Sigma^{+}\right)$radical when $J=0$.

\begin{tabular}{|c|c|c|c|c|c|}
\hline $\boldsymbol{v}$ & $H_{v}\left(\times 10^{11}\right) / \mathrm{cm}^{-1}$ & $L_{v}\left(\times 10^{17}\right) / \mathrm{cm}^{-1}$ & $M_{v}\left(\times 10^{22}\right) / \mathrm{cm}^{-1}$ & $N_{v}\left(\times 10^{27}\right) / \mathrm{cm}^{-1}$ & $O_{v}\left(\times 10^{32}\right) / \mathrm{cm}^{-1}$ \\
\hline 0 & 1.4027100 & -4.8671611 & 1.9911130 & -2.8402586 & -2.0392494 \\
\hline 1 & 1.4053343 & -5.1175272 & 1.6143796 & -3.1990403 & -2.2434658 \\
\hline 2 & 1.4053989 & -5.3917804 & 1.2293437 & -3.5529674 & -2.4676094 \\
\hline 3 & 1.4028724 & -5.6889672 & 0.83591753 & -3.9116409 & -2.7280207 \\
\hline 4 & 1.3977284 & -6.0083544 & 0.43329623 & -4.2808699 & -3.0356308 \\
\hline 5 & 1.3899449 & -6.3493767 & 0.020141443 & -4.6670931 & -3.4018218 \\
\hline 6 & 1.3795027 & -6.7116605 & -0.40542105 & -5.0774004 & -3.8395702 \\
\hline 7 & 1.3663844 & -7.0950461 & -0.84581042 & -5.5195056 & -4.3611048 \\
\hline 8 & 1.3505725 & -7.4996087 & -1.3039962 & -6.0018194 & -4.9798226 \\
\hline 9 & 1.3320486 & -7.9256784 & -1.7835120 & -6.5335708 & -5.7128176 \\
\hline 10 & 1.3107919 & -8.3738600 & -2.2884790 & -7.1248038 & -6.5756507 \\
\hline 11 & 1.2867778 & -8.8450532 & -2.8236361 & -7.7866333 & -7.5878382 \\
\hline 12 & 1.2599765 & -9.3404730 & -3.3943722 & -8.5313740 & -8.7747008 \\
\hline 13 & 1.2303516 & -9.8616728 & -4.0067932 & -9.3727447 & -10.161238 \\
\hline 14 & 1.1978589 & -10.410568 & -4.6677733 & -10.326299 & -11.793475 \\
\hline 15 & 1.1624448 & -10.989467 & -5.3850704 & -11.409132 & -13.677149 \\
\hline 16 & 1.1240448 & -11.601095 & -6.1673928 & -12.641287 & -15.864538 \\
\hline 17 & 1.0825822 & -12.248641 & -7.0245304 & -14.045587 & -18.441352 \\
\hline 18 & 1.0379661 & -12.935792 & -7.9675652 & -15.648035 & -21.437787 \\
\hline 19 & 0.99008998 & -13.666785 & -9.0089937 & -17.479025 & -24.938023 \\
\hline 20 & 0.93882954 & -14.446467 & -10.162999 & -19.573574 & -29.013928 \\
\hline
\end{tabular}

Table 6. Comparisons of vibrational levels and molecular constants with experiments and theories calculated for $\operatorname{BeF}\left(\mathrm{A}^{2} \Pi_{\mathrm{r}}\right)$ radical when $J=0$.

\begin{tabular}{|c|c|c|c|c|c|c|c|c|c|}
\hline \multirow{2}{*}{$v$} & \multicolumn{3}{|c|}{$G(v) / \mathrm{cm}^{-1}$} & \multicolumn{3}{|c|}{$B_{v} / \mathbf{c m}^{-1}$} & \multicolumn{3}{|c|}{$D_{v}\left(\times 10^{6}\right) / \mathrm{cm}^{-1}$} \\
\hline & This work & Ref. [14] & Exp. * & This work & Ref. [18] & Exp. [8] & This work & Ref. [18] & Exp. [8] \\
\hline 0 & 584.86 & 588 & 584.1 & 1.4045 & 1.4041 & 1.4115 & 8.159 & 8.152 & 8.40 \\
\hline 1 & 1741.84 & 1744 & 1739.1 & 1.3876 & 1.3866 & 1.3939 & 8.095 & 8.104 & 8.26 \\
\hline 2 & 2882.16 & 2872 & 2876.6 & 1.3709 & 1.3696 & & 8.049 & 7.953 & \\
\hline 3 & 4005.69 & 3973 & 3996.5 & 1.3545 & 1.3528 & & 7.981 & 8.015 & \\
\hline 4 & 5112.92 & 5047 & 5098.9 & 1.3380 & 1.336 & & 7.926 & 7.995 & \\
\hline 5 & 6203.86 & 6097 & 6183.7 & 1.3271 & 1.3192 & & 7.873 & 7.953 & \\
\hline 6 & 7278.62 & 7124 & 7250.9 & 1.3056 & 1.3026 & & 7.832 & 7.884 & \\
\hline 7 & 8337.27 & 8130 & 8300.6 & 1.2897 & 1.2861 & & 7.777 & 7.852 & \\
\hline 8 & 9380.07 & 9117 & 9332.7 & 1.2739 & 1.2695 & & 7.703 & 7.855 & \\
\hline 9 & 10407.47 & 10088 & 10347.3 & 1.2584 & 1.2528 & & 7.635 & 7.856 & \\
\hline 10 & 11419.76 & 11044 & 11344.3 & 1.2430 & 1.2361 & & 7.603 & 7.831 & \\
\hline 11 & 12416.79 & 12925 & 13285.6 & 1.2276 & & & 7.611 & & \\
\hline 12 & 13398.11 & 13855 & 14229.9 & 1.1212 & & & 7.634 & & \\
\hline 13 & 14363.21 & 14779 & 15156.7 & 1.1961 & & & 7.603 & & \\
\hline 14 & 15312.16 & & & 1.1807 & & & 7.451 & & \\
\hline 15 & 16246.14 & & & 1.166 & & & 7.162 & & \\
\hline 16 & 17167.19 & & & 1.1526 & & & 6.895 & & \\
\hline
\end{tabular}


Table 6. Cont.

\begin{tabular}{lllc}
\hline 17 & 18076.98 & 1.1397 & 6.919 \\
18 & 18974.86 & 1.1257 & 7.418 \\
19 & 19275.90 & 2.3327 & 6.9808 \\
20 & 19313.93 & 2.0731 & 2.9969 \\
\hline
\end{tabular}

* Taken from the reference in Reference [14].

Table 7. Vibrational levels and molecular constants for the $\mathrm{B}^{2} \Sigma^{+}$state of BeF radical.

\begin{tabular}{|c|c|c|c|}
\hline$v$ & $G(v) / \mathrm{cm}^{-1}$ & $B_{v} / \mathbf{c m}^{-1}$ & $D_{v}\left(\times 10^{6}\right) / \mathrm{cm}^{-1}$ \\
\hline 0 & 672.36 & 1.5451 & 8.263 \\
\hline 1 & 1997.79 & 1.5248 & 8.310 \\
\hline 2 & 3297.21 & 1.5042 & 8.533 \\
\hline 3 & 3565.79 & 0.3669 & 1.304 \\
\hline 4 & 3953.60 & 0.3715 & 1.377 \\
\hline 5 & 4342.89 & 0.3757 & 1.428 \\
\hline 6 & 4570.02 & 1.4833 & 8.444 \\
\hline 7 & 4733.41 & 0.3795 & 1.483 \\
\hline 8 & 5124.94 & 0.3832 & 1.533 \\
\hline 9 & 5517.25 & 0.3866 & 1.584 \\
\hline 10 & 5815.89 & 1.4621 & 8.580 \\
\hline 11 & 5910.18 & 0.3898 & 1.632 \\
\hline 12 & 6303.56 & 0.3928 & 1.686 \\
\hline 13 & 6697.25 & 0.3957 & 1.741 \\
\hline 14 & 7033.16 & 1.4399 & 8.771 \\
\hline 15 & 7091.10 & 0.3984 & 1.791 \\
\hline 16 & 7484.95 & 0.4010 & 1.849 \\
\hline 17 & 7878.67 & 0.4034 & 1.909 \\
\hline 18 & 8220.25 & 1.4176 & 8.725 \\
\hline 19 & 8272.16 & 0.4057 & 2.001 \\
\hline 20 & 8665.01 & 0.4079 & 2.056 \\
\hline
\end{tabular}

As can be seen from Table 2, the present results are in excellent agreement with the theoretical data reported in the literature. For example, the deviations from the theories [17] are of only $0.25 \%, 0.12 \%$, $0.02 \%$ and $0.23 \%$ when $v=1,3,5$ and 7 , respectively, and the deviations from the theories [18] deviate only by $0.23 \%, 0.33 \%, 0.45 \%$ and $0.64 \%$, respectively. Therefore, we can say that the present calculations are accurate. Furthermore we can conclude that the values of vibrational levels and classical turning points presented in Table 3 must be reliable.

Similar to the vibrational level spacings, there are two groups of theoretical data $[17,18]$ concerned with the inertial rotation constant $B_{v}$ and centrifugal distortion constant $D_{v}$ of $\operatorname{BeF}\left(\mathrm{X}^{2} \Sigma^{+}\right)$. For a convenient comparison with the present results, we also tabulate them in Table 4. By simple calculations, it is not difficult to find that excellent agreement exists between the present results and the theoretical data. For example for the $B_{v}$, the deviations from the theory [17] are only $0.14 \%, 0.47 \%$, and $0.51 \%$ when $v=0,2$ and 4 , respectively. As to the centrifugal distortion constant $D_{v}$, good accord also exists between the present results and the available theoretical data $[17,18]$. Therefore, the present 
calculations are accurate. According to these, the calculations of the centrifugal distortion constants presented in Table 5 should be reliable.

As can be seen from Table 6, the present results are in excellent agreement with the experiments [14]. For example, the deviations from the experiments [14] are only $0.13 \%, 0.19 \%, 0.27 \%$ and $0.38 \%$ when $v=0,2,4$ and 6, respectively. Therefore, we can say that the present calculations are accurate. For the inertial rotation constant $B_{v}$, the deviations of the present values from the experiments [8] are of $0.50 \%$ and $0.45 \%$, when $v=0$ and 1 , respectively.

To the best of our knowledge, no experimental and theoretical data of vibrational levels and molecular constants for $\operatorname{BeF}\left(\mathrm{B}^{2} \Sigma^{+}\right)$has been found in the literature. However, according to the remarkable agreement between the present spectroscopic parameters and the available experiments $[8,11]$, we have reasons to believe that the results collected in Tables 5 are accurate.

\section{Conclusions}

In the present work, the PECs of $\mathrm{X}^{2} \Sigma^{+}, \mathrm{A}^{2} \Pi_{\mathrm{r}}$ and $\mathrm{B}^{2} \Sigma^{+}$states of BeF radical have been investigated by the MRCI approach with large correlation-consistent basis sets, cc-pV5Z for Be and aug-cc-pV6Z for F. Based on the PECs of these three states, the spectroscopic parameters and molecular constants are determined in the present work, and the values are in excellent agreement with the experimental data. With the PECs of these states determined at the MRCI level of theory, the vibrational levels, inertial rotation and centrifugal distortion constants are predicted, and the classical turning points are also calculated for the $\mathrm{X}^{2} \Sigma^{+}$state when $J=0$. On the whole, comparison with the available experiments and theories shows that the present calculations are both reliable and accurate.

\section{Acknowledgements}

This work was supported by the National Natural Science Foundation of China (Grant No. 10874064), the Natural Science Foundation of Henan Province in China (Grant No. 2008A140008), and the Key Teachers Foundation of Henan Province in China (Grant No. 2008043).

\section{References}

1. Popkie, H.E.; Henneker, W.H. Theoretical electronic transition probabilities in diatomic molecules II. 13-electron sequence. J. Chem. Phys. 1971, 55, 617-628.

2. Mezey, P.G.; Bernardi, F.; Csizmadia, I.G.; Strausz, O.P. Ab initio MO calculation of the $\operatorname{Be}\left(2^{3} \mathrm{P}\right)$ $+\mathrm{CH}_{4}$ reaction. Chem. Phys. Lett. 1978, 59, 117-120.

3. Kuntz, P.J.; Roach, A.C. A diatomics-in-molecules case study on the system $\mathrm{Be}+\mathrm{HF} \rightarrow \mathrm{BeF}+$ H.I. Bonding models and the use of valence bond information. J. Chem. Phys. 1981, 74, 3420-3434.

4. Kobus, J.; Moncrieff, D.; Wilson, S. Comparison of the electric moments obtained from finite basis set and finite-difference Hartree-Fock calculations for diatomic molecules. Phys. Rev. A 2000, 62, 062503:1-062503:9.

5. Guan, J.; Casida, M.E.; Salahub, D.R. Time-dependent density-functional theory investigation of excitation spectra of open-shell molecules. J. Mol. Struct. (Theochem.) 2000, 527, 229-244. 
6. Lörinczy, D.; Kiss, M.; Belagyi, J. DSC and EPR study on AMP.PNP, BeF $\mathrm{x}_{\mathrm{x}}$ and $\mathrm{AlF}_{4}$ containing myosin nucleotide complexes. J. Therm. Anal. Calorim. 2003, 72, 565-572.

7. Hildenbrand, D.L.; Murad, E. Mass-spectrometric determination of the dissociation energy of beryllium monofluoride. J. Chem. Phys. 1966, 44, 1524-1529.

8. Walker, T.E.H.; Barrow, R.F. The $\mathrm{A}^{2} \prod-\mathrm{X}^{2} \Sigma^{+}$system of BeF. J. Phys. B 1969, 2, 102-106.

9. Farber, M.; Srivastava, R.D. Dissociation energies of $\mathrm{BeF}$ and $\mathrm{BeCl}$ and the heat of formation of BeClF. J. Chem. Soc. Faraday Trans. 1974, 70, 1581-1589.

10. Herzberg, G. Molecular Spectra and Molecular Structure, Spectra and Diatomic Molecules; Van Nostrand Reinhold: New York, NY, USA, 1950; volume I, p. 402.

11. Huber, K.P.; Herzberg, G. Molecular Spectra and Molecular Structure, Constants of Diatomic Molecules; Van Nostrand Reinhold: New York, NY, USA, 1979; volume IV, p. 76.

12. Roach, A.C.; Kuntz, P.J. Bonding in BeF and a consequent unusual progression of vibrational spacings. Mol. Phys. 1982, 45, 853-856.

13. Partridge, H.; Bauschlicher, C.W.; Langhoff, S.R. The dissociation energy of ionic molecules; Selected oxides and fluorides: $\mathrm{LiO}, \mathrm{LiF}, \mathrm{BeO}, \mathrm{BeF}, \mathrm{MgF}, \mathrm{CaF}$ and SrF. Chem. Phys. Lett. 1984, $109,446-449$.

14. Marian, C.M. Spin-orbit splitting of the $A^{2} \Pi$ and $D^{2} \Pi$ states of BeF by ab initio MRD CI calculations. Chem. Phys. 1985, 100, 13-19.

15. Langhoff, S.R.; Bauschlicher, C.W.; Partridge, H. Theoretical dissociation energies for the alkali and alkaline-earth monofluorides and monochlorides. J. Chem. Phys. 1986, 84, 1687-1695.

16. Langhoff, S.R.; Bauschlicher, C.W.; Partridge, H.; Ahlrichs, R. Theoretical study of the dipole moments of selected alkaline-earth halides. J. Chem. Phys. 1986, 84, 5025-5031.

17. Machado, F.B.C.; Ornellas, F.R. A theoretical study of the BeF molecule in the $\mathrm{X}^{2} \Sigma^{+}$state. Mol. Phys. 1989, 67, 1129-1140.

18. Ornellas, F.R.; Machado, F.B.C.; Roberto-neto, O. A theoretical study of the molecules BeF and $\mathrm{BeF}^{+}$in their lowest-lying electronic states. Mol. Phys. 1992, 77, 1169-1185.

19. Li, G.P.; Hamilton, I.P. Dimers of alkaline earth metal halide radicals, $(\mathrm{MX})_{2}(\mathrm{M}=\mathrm{Be}, \mathrm{Mg}, \mathrm{Ca}$; $\mathrm{X}=\mathrm{F}, \mathrm{Cl}$ ): A theoretical study. J. Chem. Phys. 2001, 114, 1534-1538.

20. Pelegrini, M.; Vivacqua, C.S.; Roberto-Neto, O.; Ornellas, F.R.; Machado, F.B.C. Radiative transition probabilities and lifetimes for the band systems $A^{2} \Pi-X^{2} \Sigma^{+}$of the isovalent molecules BeF, MgF and CaF. Braz. J. Phys. 2005, 35, 950-956.

21. Walker, T.E.H.; Richards, W.G. The nature of the first excited electronic state in BeF. Proc. Phys. Soc. 1967, 92, 285-290.

22. Werner, H.-J.; Knowles, P.J. A second order multiconfiguration SCF procedure with optimum convergence. J. Chem. Phys. 1985, 82, 5053-5063.

23. Knowles, P.J.; Werner, H.-J. An efficient second-order MC SCF method for long configuration expansions. Chem. Phys. Lett. 1985, 115, 259-267.

24. Werner, H.-J.; Knowles, P.J. An efficient internally contracted multiconfiguration-reference configuration interaction method. J. Chem. Phys. 1988, 89, 5803-5814.

25. Knowles, P.J.; Werner, H.-J. An efficient method for the evaluation of coupling coefficients in configuration interaction calculations. Chem. Phys. Lett. 1988, 145, 514-522. 
26. Kendall, R.A.; Dunning, T.H.; Harrison, R.J. Electron affinities of the first-row atoms revisited. Systematic basis sets and wave functions. J. Chem. Phys. 1992, 96, 6796-6806.

27. Dunning, T.H. Gaussian basis sets for use in correlated molecular calculations. I. The atoms boron through neon and hydrogen. J. Chem. Phys. 1989, 90, 1007-1023.

28. Woon, D.E.; Dunning, T.H. Gaussian basis sets for use in correlated molecular calculations. III. The atoms aluminum through argon. J. Chem. Phys. 1993, 98, 1358-1371.

29. Karlström, G.; Lindh, R.; Malmqvist, P.-Å.; Roos, B.O.; Ryde, U.; Veryazov, V.; Widmark, P.-O.; Cossi, M.; Schimmelpfennig, B.; Neogrady, P.; et al. MOLCAS: A program package for computational chemistry. Comp. Mater. Sci. 2003, 28, 222-239.

30. Shi, D.H.; Zhang, X.N.; Sun, J.F.; Zhu, Z.L. MRCI study on spectroscopic and molecular properties of $\mathrm{B}^{1} \Delta_{\mathrm{g}}, \mathrm{B}^{\prime 1} \Sigma_{\mathrm{g}}{ }^{+}, \mathrm{C}^{1} \Pi_{\mathrm{g}}, \mathrm{D}^{1} \Sigma_{\mathrm{u}}{ }^{+}, \mathrm{E}^{1} \Sigma_{\mathrm{g}}{ }^{+}$and $1^{1} \Delta_{\mathrm{u}}$ electronic states of the $\mathrm{C}_{2}$ radical. Mol. Phys. 2011, $109,1453-1465$.

31. Zhang, X.N.; Shi, D.H.; Sun, J.F.; Zhu, Z.L. MRCI study on spectroscopic and molecular properties of $\mathrm{X}^{2} \Pi_{\mathrm{g}}, a^{4} \Pi_{\mathrm{u}}, \mathrm{A}^{2} \Pi_{\mathrm{u}}, b^{4} \Sigma_{\mathrm{g}}^{-}, \mathrm{D}^{2} \Delta_{\mathrm{g}}$ and $\mathrm{B}^{2} \Sigma_{\mathrm{g}}^{-}$electronic states of $\mathrm{O}_{2}^{+}$ion. Mol. Phys. 2011, 109, 1627-1638.

32. Shi, D.H.; Liu, H.; Sun, J.F.; Liu, Y.F.; Zhu, Z.L. MRCI study on spectroscopic and molecular properties of four low-lying electronic states of the BO radical. J. Mol. Spectrosc. 2010, 264, $55-60$.

33. Shi, D.H.; Li, W.T.; Zhang, X.N.; Sun, J.F.; Liu, Y.F.; Zhu, Z.L.; Wang, J.M. Effects on spectroscopic properties for several low-lying electronic states of CS molecule by core-valence correlation and relativistic corrections. J. Mol. Spectrosc. 2011, 266, 27-36.

34. Shi, D.H.; Liu, H.; Sun, J.F.; Zhu, Z.L.; Liu, Y.F. Effects on spectroscopic parameters of several low-lying electronic states of GeS by core-valence correlation and relativistic corrections. J. Mol. Spectrosc. 2011, 269, 143-150.

35. Shi, D.H.; Liu, H.; Sun, J.F.; Zhu, Z.L.; Liu, Y.F. Spectroscopic and molecular properties of 14 selected electronic states of $\mathrm{Si}_{2}$ molecule. J. Quant. Spectrosc. Radiat. Transf. 2011, 112, 2567-2583.

36. Werner, H.-J.; Knowles, P.J.; Lindh, R.; Manby, F.R.; Schütz, M.; Celani, P.; Korona, T.; Mitrushenkov, A.; Rauhut, G.; Adler, T.B.; et al. MOLPRO 2008, version 2008.1, a package of $a b$ initio programs; Cardiff University: Cardiff, UK, 2008.

(C) 2012 by the authors; licensee MDPI, Basel, Switzerland. This article is an open access article distributed under the terms and conditions of the Creative Commons Attribution license (http://creativecommons.org/licenses/by/3.0/). 